\title{
Call for Papers Issue 1/2020
}

\section{High Performance Business Computing}

\author{
Guido Schryen • Natalia Kliewer • Andreas Fink
}

Published online: 10 July 2018

(C) Springer Fachmedien Wiesbaden GmbH, part of Springer Nature 2018

\section{Special Issue}

High Performance Business Computing (HPBC) is the application of models, methodologies, tools and technologies of High Performance Computing (HPC) (e.g., Hager and Wellein 2010) to business problems. While its neighbor discipline, High Performance Technical Computing, has already found entrance into a variety of scientific disciplines, including meteorology, engineering, bio sciences, physics, chemistry and mathematics, the fields of management science (MS) and information systems research (ISR) have largely neglected to start exploiting the potentials that HPC offer for business problems and addressing the need of modern business computing for high computing power and capability to deal with huge volumes of data.

The scientific development and practical deployment of HPBC is useful, if not even necessary, for essentially three reasons: First, the fields of MS and ISR become increasingly computational with the advent of large volumes of data and the pervasion of computing resources in business, scientific and

Prof. Dr. G. Schryen $(\bowtie)$

Universität Paderborn, Warburger Straße 100, 33098 Paderborn, Germany

e-mail: guido.schryen@upb.de

URL: https://wiwi.uni-paderborn.de/dep3/schryen/

Prof. Dr. N. Kliewer

Freie Universität Berlin, Garystr. 21, 14195 Berlin, Germany

e-mail: natalia.kliewer@fu-berlin.de

URL: http://www.wiwiss.fu-berlin.de/kliewer

Prof. Dr. A. Fink

Institut für Informatik, Helmut-Schmidt-Universität Hamburg,

Holstenhofweg 85, 22043 Hamburg, Germany

e-mail: andreas.fink@hsu-hamburg.de

URL: https://www.hsu-hh.de/ifi/team/fink social environments. Expectations and business opportunities that result from these developments include the potentials of real-time decision making (e.g., for IT security infrastructure protection and financial stock trading), solving computationally hard optimization problems (e.g., in production and logistics) (e.g., Gendron and Crainic 1994; Aldasoro et al. 2017), and analyzing huge volumes of data acquired from sensors, mobile phones, and social networks (e.g., for social network analysis, fraud detection and business analytics) (e.g., Wang et al. 2016; Zhang et al. 2016). The analysis of such data has already started attracting new machine learning approaches. Second, HPC infrastructures, tools, libraries and services are becoming commodity goods offered by computing centers of universities, public cloud providers (e.g., as part of Amazon Web services), and open source communities (e.g., Apache Hadoop framework). Third, the end of exponential growth in single processor core performance, which has been a key driver of the economic and technology-advancement cycles over the past decades, marks the end of the dominance of the single microprocessor in computing, and the era of sequential computing must give way to a new era in which parallelism is at the forefront (Fuller and Millet 2011). However, sequential programs (as models and methodologies in general) will not benefit from increases in processor performance that stem from the use of parallel processing capabilities.

This special issue accounts for the aforementioned challenges of HPBC by calling for quantitative and computational studies in the areas of business optimization, data and business analytics, and machine learning. Of particular interest are studies related to the development and computational validation of parallel or distributed (1) (exact or heuristic) algorithms for optimization problems, (2) data analysis algorithms for business analytics, and (3) machine learning algorithms for business analytics. 


\section{Submission}

Authors are asked to submit their papers online under the category "BISE High Performance Business Computing" by 2019-03-01 via Springer's submission system Editorial Manager (http://www.editorialmanager.com/buis/). All papers must follow the typing and formatting instructions for Business \& Information Systems Engineering (BISE) available at http://www.bise-journal.com/?page_id=18. In particular, manuscripts should not exceed 50,000 characters (discounting 5000 characters for each figure/table).

Submitted papers will undergo a double-blind review process and be refereed by at least three domain experts according to quality, originality, relevance, and scientific rigor.

\section{Schedule}

\begin{tabular}{ll}
\hline Paper submission due: & 1 March 2019 \\
Notification of authors: & 2 May 2019 \\
Revisions due: & 1 July 2019 \\
Notification of authors: & 15 August 2019 \\
Completion of a second revision (if needed): & 19 September 2019 \\
Anticipated publication date: & February 2020 \\
\hline
\end{tabular}

\section{References}

Aldasoro U, Escudero LF, Merino M, Perez G (2017) A parallel branch-and-fix coordination based matheuristic algorithm for solving large sized multistage stochastic mixed $0-1$ problems. Eur J Oper Res 258:590-606

Fuller SH, Millet LI (2011) The future of computing performance: game over or next level? In: Fuller SH, Millett LI (eds) National research council of the national academies. National Academies Press, Washington, D.C.

Gendron B, Crainic TG (1994) Parallel branch-and-bound algorithms: survey and synthesis. Oper Res 42(6):1042-1066

Hager G, Wellein G (2010) Introduction to high performance computing for scientists and engineers. CRC Press, Boca Raton

Wang Q, Li B, Wang P, Yang J (2016) Using TB-sized data to understand multi-device advertising. In: Proceedings of the international conference on information systems, Dublin

Zhang K, Bhattacharyya S, Ram S (2016) Large-scale network analysis for online social brand advertising. MIS Q 40(4):849-868 